\title{
Synchronization and Antisynchronization of N-Coupled Complex Permanent Magnet Synchronous Motor Systems with Ring Connection
}

\author{
Cuimei Jiang and Shutang Liu \\ College of Control Science and Engineering, Shandong University, Jinan 250061, China \\ Correspondence should be addressed to Shutang Liu; stliu@sdu.edu.cn
}

Received 7 July 2016; Revised 18 September 2016; Accepted 20 September 2016; Published 26 January 2017

Academic Editor: Roberto Natella

Copyright (C) 2017 C. Jiang and S. Liu. This is an open access article distributed under the Creative Commons Attribution License, which permits unrestricted use, distribution, and reproduction in any medium, provided the original work is properly cited.

\begin{abstract}
This paper discusses synchronization and antisynchronization of $N$-coupled complex permanent magnet synchronous motors systems with ring connection. Based on the direct design method and antisymmetric structure, the appropriate controllers are designed to ensure the occurrence of synchronization and antisynchronization in an array of $N$-coupled general complex chaotic systems described by a unified mathematical expression with ring connection. The proposed method is flexible and is suitable both for design and for implementation in practice. Numerical results are plotted to show the rapid convergence of errors to zero and further verify the effectiveness and feasibility of the theoretical scheme.
\end{abstract}

\section{Introduction}

Since Fowler et al. [1] were the first to investigate the complex Lorenz equations, complex systems have played an important role in a variety of industrial fields. For example, complex systems can provide an excellent instrument for the description of various physical phenomena, such as detuned laser systems, amplitudes of electromagnetic fields, and thermal convection of liquid flows [2-4]. Another example is when synchronization of complex chaotic systems is used to transmit information in the secure communications, where complex variables (doubling number of real variables) increase the message contents and enhance security of the transmitted information. Hence, synchronization of complex chaotic systems has aroused great interest and been a hot interdisciplinary topic in nonlinear research during the last few decades [5-9]. Some synchronization schemes of real chaotic systems were extended in the complex field, such as complete synchronization [10], antisynchronization [11, 12], projective synchronization [13], and lag synchronization [14]. Additionally, there exist many synchronization schemes of complex chaotic systems, including complex complete synchronization [15], complex projective synchronization [16], complex modified projective synchronization [17, 18], and complex function projective synchronization [7], just to enumerate a few examples.

With the mentioned above, most of the works mainly focus on the usual drive-response synchronization within one drive system and one response system; there is very little concern on synchronization of multiple complex chaotic systems. As a matter of fact, synchronization of multiple complex systems can enhance better the security and lead a more bright future in communications than the usual synchronization schemes. Therefore, many researchers have devoted a great deal of time and efforts to study and analyze synchronization of multiple complex chaotic systems. For instance, Zhou et al. [19] investigated the combination synchronization problem in three identical and different hyperchaotic complex systems by means of two real scaling diagonal matrices. As introduced in [20], combination-combination synchronization among different nonlinear complex chaotic systems was realized by designing proper controllers. Sun et al. [21] made an excellent effort for examining combination complex synchronization of three identical hyperchaotic complex systems via two 


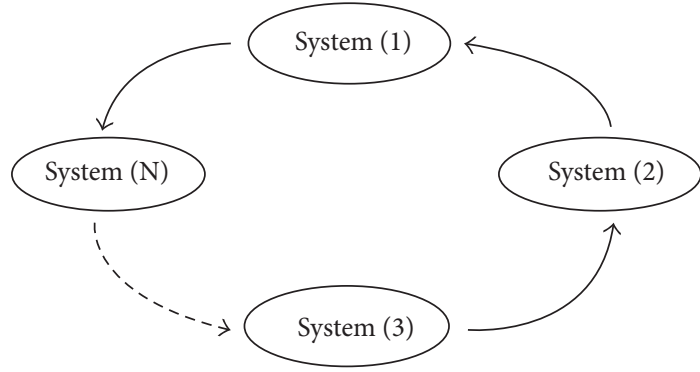

Figure 1: Diagram of coupled complex chaotic systems with ring connection.

complex scaling diagonal matrices. Noticeably, Jiang and Liu [22] analyzed combination complex synchronization of real chaos and complex chaos with different dimensions, namely, generalized combination complex synchronization. Furthermore, a novel adaptive generalized combination complex synchronization scheme for different real and complex nonlinear systems with unknown parameters was reported in a recent literature [23].

To the best of our knowledge, the aforementioned results on synchronization of multiple complex chaotic systems did not consider the case that multiple complex chaotic systems are coupled in a ring structure as shown in Figure 1. In this chaotic model, the state variables of the first complex chaotic system couple the $N$ th system, and the state variables of the second system couple the first,... analogically, until the state variables of the $N$ th system couple those of the $(N-1)$ th. Thus, the ring structure formed among multiple complex chaotic systems makes them correlative. Consequently, it is much more attractive and challenging for researchers to explore various synchronization schemes of multiple coupled complex chaotic systems with ring connection. Moreover, the previous attention was drawn for the research on synchronization of multiple coupled real chaotic systems with ring connection, such as [24-29] to name a few. To date, the study has rarely been explored to achieve synchronization of multiple coupled complex chaotic systems with ring connection.

Up till now, there are many approaches for realizing chaos synchronization [30-32]. It should be mentioned that activation control is one of the most important approaches for synchronizing chaotic systems. By means of activation method, controllers can be constructed by canceling all nonlinear terms in original systems, and further synchronization of chaotic systems is converted to a linear problem. Meanwhile, the design procedure of sliding mode method is complex and the controller obtained by this method is not simple enough for many systems, while in [27-29, 33] authors presented the direct design approach based on antisymmetric structure to realize synchronization of chaotic systems with real variables. This direct design method has two advantages: (i) it presents an easy procedure for choosing proper controllers in chaos synchronization; (ii) it constructs simple controllers easily to implement. Therefore, in this paper, for $\mathrm{N}$-coupled complex chaotic systems with ring connection, we utilize the direct method based on antisymmetric structure to investigate chaos synchronization.

On the other hand, permanent magnet synchronous motor (PMSM) has direct applications in many areas especially for industrial applications in low-medium power range, since it has excellent features such as simple structure, high torque-to-inertia ratio, high torque-to-weight ratio, and low manufacturing cost. Therefore, a considerable amount of research has been devoted to study synchronization and control of the PMSM system [34-38]. However, so far, there are few papers discussing dynamical properties of the PMSM system with complex variables $[39,40]$. Meanwhile, complex voltage and current exist widely in motor systems which result in more complex and richer dynamical dynamics in practice. Therefore, it is of practical significance to investigate the complex PMSM system.

Motivated by the above discussions, in this paper, we concentrate on synchronization and antisynchronization of $N$-coupled complex PMSM systems with ring connection for the first time. By virtue of the direct method, the general controllers are constructed to transform the error system into a nonlinear system with special antisymmetric structure and achieve synchronization and antisynchronization of $\mathrm{N}$ coupled general complex chaotic systems. What is more, this proposed synchronization scheme is simple but efficacious.

\section{System Descriptions}

Consider a PMSM system in field-oriented rotor which takes the following form:

$$
\begin{aligned}
& \frac{d i_{d}}{d t}=\frac{\left(-R_{1} i_{d}+\omega L_{q} i_{q}+u_{d}\right)}{L_{d}}, \\
& \frac{d i_{q}}{d t}=\frac{\left(-R_{1} i_{q}+\omega L_{d} i_{d}+u_{q}-\omega \psi_{r}\right)}{L_{q}}, \\
& \frac{d \omega}{d t}=\frac{\left(n_{p} \psi_{r} i_{q}+n_{p}\left(L_{d}-L_{q}\right) i_{d} i_{q}-T_{L}-\beta \omega\right)}{J},
\end{aligned}
$$

where $i_{d}, i_{q}$, and $\omega$ are the state variables which represent currents and motor angular frequency, respectively; $u_{d}$ and $u_{q}$ are the direct-axis stator and quadrature-axis stator voltage components, respectively; $J$ is the polar moment of inertia; $T_{L}$ is the external load torque; $\beta$ is the viscous damping coefficient; $R_{1}$ is the stator resistance; $L_{d}$ and $L_{q}$ are the directaxis and quadrature-axis inductances, respectively; $\psi_{r}$ is rotor flux; and $n_{p}$ is pole-pairs.

When the air gap is even and the motor operates with no load and power outage, the dimensionless equations of a PMSM system can be expressed as follows:

$$
\begin{aligned}
& \dot{x}_{1}=a\left(x_{2}-x_{1}\right), \\
& \dot{x}_{2}=b x_{1}-x_{2}-x_{1} x_{3}, \\
& \dot{x}_{3}=x_{1} x_{2}-x_{3},
\end{aligned}
$$




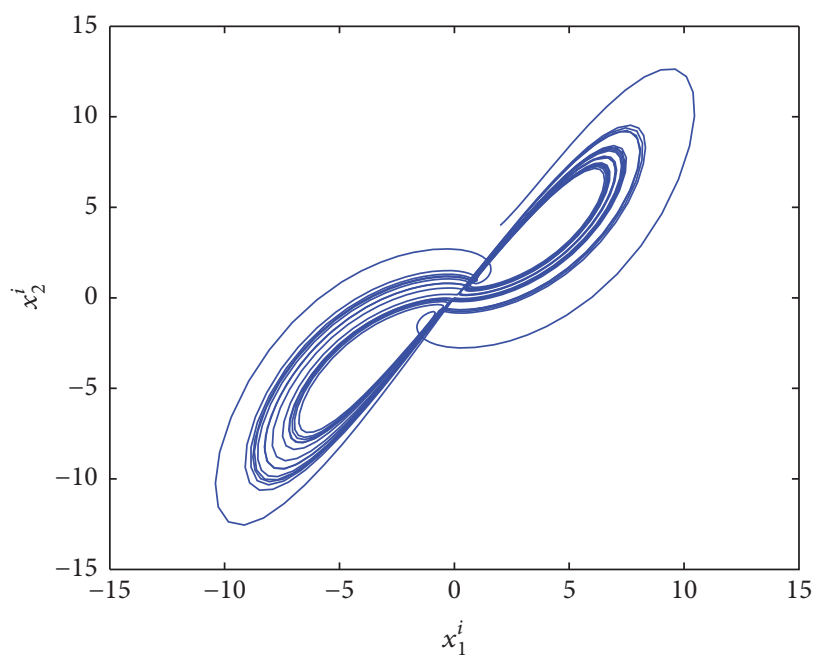

(a)

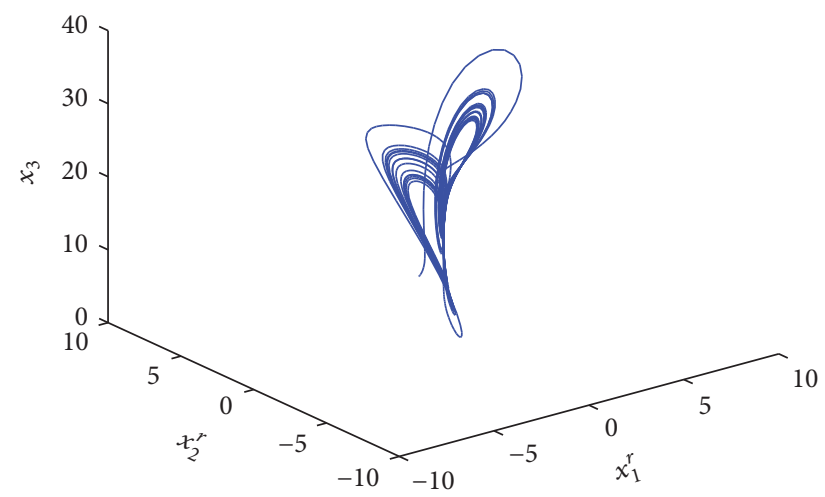

(b)

FIGURE 2: Chaotic behavior and attractors of the complex PMSM system (3) in different phase planes and projections.

where $a$ and $b$ are parameters. Considering that current in system (1) is plural and variables $x_{1}$ and $x_{2}$ in system (2) are complex numbers, Wang and Zhang [39] introduced a complex PMSM system as follows:

$$
\begin{aligned}
& \dot{x}_{1}=a\left(x_{2}-x_{1}\right), \\
& \dot{x}_{2}=b x_{1}-x_{2}-x_{1} x_{3}, \\
& \dot{x}_{3}=\frac{1}{2}\left(\bar{x}_{1} x_{2}+x_{1} \bar{x}_{2}\right)-x_{3},
\end{aligned}
$$

where $x_{1}$ and $x_{2}$ are complex variables and $\bar{x}_{1}$ and $\bar{x}_{2}$ are conjugate complex numbers of $x_{1}$ and $x_{2}, j=\sqrt{-1}$. When $a=11$ and $b=20$, system (3) is chaotic and the phase portraits are shown in Figure 2.

Authors studied some basic dynamical properties of the complex PMSM system (3) in [39, 40]. But there are many properties of the complex PMSM system remaining to be uncovered. In the sequel, we will discuss synchronization and antisynchronization of $N$-coupled complex PMSM systems with ring connection.

The main construct of the rest of the paper is organized as follows. Section 3 investigates synchronization and antisynchronization of $\mathrm{N}$-coupled general complex chaotic systems described by a unified mathematical expression with ring connection. In Section 4, the complex controllers are provided to realize synchronization and antisynchronization of 4-coupled complex PMSM systems with ring connection. Finally, Section 5 gives some concluding remarks.

\section{General Method for Synchronization and Antisynchronization of $N$-Coupled Complex Chaotic Systems}

A general form of $N$-coupled complex chaotic systems with ring connection can be configured as

$$
\dot{X}_{1}=A_{1} X_{1}+G_{1}\left(X_{1}\right)+Q_{1}\left(X_{N}-X_{1}\right),
$$

$$
\begin{gathered}
\dot{X}_{2}=A_{2} X_{2}+G_{2}\left(X_{2}\right)+Q_{2}\left(X_{1}-X_{2}\right), \\
\vdots \\
\dot{X}_{N}=A_{N} X_{N}+G_{N}\left(X_{N}\right)+Q_{N}\left(X_{N-1}-X_{N}\right),
\end{gathered}
$$

where $X_{1}, X_{2}, \ldots, X_{N} \in \mathbb{C}^{n}(N>2)$ are complex state vectors of chaotic systems and $X_{k}=X_{k}^{r}+j X_{k}^{i} ; A_{1}, A_{2}, \ldots, A_{N} \in$ $\mathbb{R}^{n \times n}$ are constant matrices $\left(A_{k} \neq A_{l}, k \neq l\right) ; G_{k}\left(X_{k}\right)$ are the continuous complex nonlinear functions $\left(G_{k} \neq G_{l}, k \neq l\right)$; $Q_{k}=\operatorname{diag}\left(q_{k 1}, q_{k 2}, \ldots, q_{k n}\right)$ are $n$-dimensional real diagonal matrices; and $q_{k l} \geq 0$ are the ideal gains which represent the coupled parameters $(k=1,2, \ldots, N ; l=1,2, \ldots, n)$.

Suppose that the first system is chosen as the aim system, and add the controllers to the remaining systems. Thus system (4) can be rewritten as

$$
\begin{gathered}
\dot{X}_{1}=A_{1} X_{1}+G_{1}\left(X_{1}\right)+Q_{1}\left(X_{N}-X_{1}\right), \\
\dot{X}_{2}=A_{2} X_{2}+G_{2}\left(X_{2}\right)+Q_{2}\left(X_{1}-X_{2}\right)+U_{1}, \\
\dot{X}_{3}=A_{3} X_{3}+G_{3}\left(X_{3}\right)+Q_{3}\left(X_{2}-X_{3}\right)+U_{2},
\end{gathered}
$$

$$
\dot{X}_{N}=A_{N} X_{N}+G_{N}\left(X_{N}\right)+Q_{N}\left(X_{N-1}-X_{N}\right)+U_{N-1}
$$

The synchronization error vector is defined as $e_{k}=X_{k+1}-$ $\delta X_{k}(k=1,2, \ldots, N-1 ; \delta=1,-1)$. Apparently, if $\delta=1$, then the error system $e_{k}$ can degenerate into the complete synchronization error system. And if $\delta=-1$, then the error system $e_{k}$ can degenerate into the antisynchronization error 
system. Taking into account system (5), we have the following error dynamical system as

$$
\begin{aligned}
\dot{e}(t) & =\dot{e}^{r}(t)+j \dot{e}^{i}(t) \\
& =\Sigma\left(\begin{array}{c}
e_{1} \\
e_{2} \\
\vdots \\
e_{N-1}
\end{array}\right) \\
& +\left(\begin{array}{c}
\left(\delta\left(A_{2}-A_{1}\right)+(1-\delta) Q_{2}-\left(\delta^{N}-\delta\right) Q_{1}\right) X_{1}+G_{2}\left(X_{2}\right)-\delta G_{1}\left(X_{1}\right)+U_{1} \\
\left(\delta\left(A_{3}-A_{2}\right)+(\delta-1)\left(Q_{2}-Q_{3}\right)\right) X_{2}+G_{3}\left(X_{3}\right)-\delta G_{2}\left(X_{2}\right)+U_{2}-\delta U_{1} \\
\vdots \\
\left(\delta\left(A_{N}-A_{N-1}\right)+(\delta-1)\left(Q_{N-1}-Q_{N}\right)\right) X_{N-1}+G_{N}\left(X_{N}\right)-\delta G_{N-1}\left(X_{N-1}\right)+U_{N-1}-\delta U_{N-2}
\end{array}\right)
\end{aligned}
$$

where

$$
\Sigma=\left(\begin{array}{cccccc}
A_{2}-\delta^{N-1} Q_{1}-Q_{2} & -\delta^{N-2} Q_{1} & -\delta^{N-3} Q_{1} & \cdots & -\delta^{2} Q_{1} & -\delta Q_{1} \\
Q_{2} & A_{3}-Q_{3} & 0 & \cdots & 0 & 0 \\
\vdots & \vdots & \vdots & \ddots & \vdots & \vdots \\
0 & 0 & 0 & \cdots & Q_{N-1} & A_{N}-Q_{N}
\end{array}\right)
$$

Furthermore, it is not difficult to obtain that

$$
\begin{aligned}
& \left(\begin{array}{c}
\dot{e}_{1}^{r} \\
\dot{e}_{2}^{r} \\
\vdots \\
\dot{e}_{N-1}^{r}
\end{array}\right)=\Sigma\left(\begin{array}{c}
e_{1}^{r} \\
e_{2}^{r} \\
\vdots \\
e_{N-1}^{r}
\end{array}\right) \\
& +\left(\begin{array}{c}
\left(\delta\left(A_{2}-A_{1}\right)+(1-\delta) Q_{2}-\left(\delta^{N}-\delta\right) Q_{1}\right) X_{1}^{r}+G_{2}^{r}\left(X_{2}\right)-\delta G_{1}^{r}\left(X_{1}\right)+U_{1}^{r} \\
\left(\delta\left(A_{3}-A_{2}\right)+(\delta-1)\left(Q_{2}-Q_{3}\right)\right) X_{2}^{r}+G_{3}^{r}\left(X_{3}\right)-\delta G_{2}^{r}\left(X_{2}\right)+U_{2}^{r}-\delta U_{1}^{r} \\
\vdots \\
\left(\delta\left(A_{N}-A_{N-1}\right)+(\delta-1)\left(Q_{N-1}-Q_{N}\right)\right) X_{N-1}^{r}+G_{N}^{r}\left(X_{N}\right)-\delta G_{N-1}^{r}\left(X_{N-1}\right)+U_{N-1}^{r}-\delta U_{N-2}^{r}
\end{array}\right) \\
& \left(\begin{array}{c}
\dot{e}_{1}^{i} \\
\dot{e}_{2}^{i} \\
\vdots \\
\dot{e}_{N-1}^{i}
\end{array}\right)=\Sigma\left(\begin{array}{c}
e_{1}^{i} \\
e_{2}^{i} \\
\vdots \\
e_{N-1}^{i}
\end{array}\right)
\end{aligned}
$$




$$
+\left(\begin{array}{c}
\left(\delta\left(A_{2}-A_{1}\right)+(1-\delta) Q_{2}-\left(\delta^{N}-\delta\right) Q_{1}\right) X_{1}^{i}+G_{2}^{i}\left(X_{2}\right)-\delta G_{1}^{i}\left(X_{1}\right)+U_{1}^{i} \\
\left(\delta\left(A_{3}-A_{2}\right)+(\delta-1)\left(Q_{2}-Q_{3}\right)\right) X_{2}^{i}+G_{3}^{i}\left(X_{3}\right)-\delta G_{2}^{i}\left(X_{2}\right)+U_{2}^{i}-\delta U_{1}^{i} \\
\vdots \\
\left(\delta\left(A_{N}-A_{N-1}\right)+(\delta-1)\left(Q_{N-1}-Q_{N}\right)\right) X_{N-1}^{i}+G_{N}^{i}\left(X_{N}\right)-\delta G_{N-1}^{i}\left(X_{N-1}\right)+U_{N-1}^{i}-\delta U_{N-2}^{i}
\end{array}\right) .
$$

Thus the essential goal of this paper is to design complex controllers $U_{k}=U_{k}^{r}+j U_{k}^{i}(k=1,2, \ldots, N-1)$ such that the error dynamical error $e(t)$ tends to zero as $t \rightarrow \infty$; namely,

$$
\begin{aligned}
& \lim _{t \rightarrow \infty}\left\|e^{r}(t)\right\|=0, \\
& \lim _{t \rightarrow \infty}\left\|e^{i}(t)\right\|=0,
\end{aligned}
$$

and then

$$
\lim _{t \rightarrow \infty}\|e(t)\|=0,
$$

where $\|\cdot\|$ is the Euclidean norm. This implies that synchronization and antisynchronization of $\mathrm{N}$-coupled complex chaotic systems are realized.

In what follows, applying the direct method based on antisymmetric structure, we investigate synchronization and antisynchronization of $\mathrm{N}$-coupled chaotic systems with complex variables.

First, the controllers $U_{k}^{r}$ and $U_{k}^{i}(k=1,2, \ldots, N-1)$ are chosen as

$$
\begin{aligned}
U_{1}^{r} & =V_{1}^{r} \\
& -\left(\delta\left(A_{2}-A_{1}\right)+(1-\delta) Q_{2}-\left(\delta^{N}-\delta\right) Q_{1}\right) X_{1}^{r} \\
& -G_{2}^{r}\left(X_{2}\right)+\delta G_{1}^{r}\left(X_{1}\right), \\
U_{2}^{r} & =V_{2}^{r}-\left(\delta\left(A_{3}-A_{2}\right)+(\delta-1)\left(Q_{2}-Q_{3}\right)\right) X_{2}^{r} \\
& -G_{3}^{r}\left(X_{3}\right)+\delta G_{2}^{r}\left(X_{2}\right)+\delta U_{1}^{r}, \\
& \vdots \\
U_{N-1}^{r} & =V_{N-1}^{r} \\
& -\left(\delta\left(A_{N}-A_{N-1}\right)+(\delta-1)\left(Q_{N-1}-Q_{N}\right)\right) X_{N-1}^{r} \\
& -G_{N}^{r}\left(X_{N}\right)+\delta G_{N-1}^{r}\left(X_{N-1}\right)+\delta U_{N-2}^{r}, \\
U_{1}^{i} & =V_{1}^{i} \\
& -\left(\delta\left(A_{2}-A_{1}\right)+(1-\delta) Q_{2}-\left(\delta^{N}-\delta\right) Q_{1}\right) X_{1}^{i} \\
& -G_{2}^{i}\left(X_{2}\right)+\delta G_{1}^{i}\left(X_{1}\right), \\
U_{2}^{i} & =V_{2}^{i}-\left(\delta\left(A_{3}-A_{2}\right)+(\delta-1)\left(Q_{2}-Q_{3}\right)\right) X_{2}^{i} \\
& -G_{3}^{i}\left(X_{3}\right)+\delta G_{2}^{i}\left(X_{2}\right)+\delta U_{1}^{i},
\end{aligned}
$$

$$
\begin{aligned}
& U_{N-1}^{i}=V_{N-1}^{i} \\
& \quad-\left(\delta\left(A_{N}-A_{N-1}\right)+(\delta-1)\left(Q_{N-1}-Q_{N}\right)\right) X_{N-1}^{i} \\
& \quad-G_{N}^{i}\left(X_{N}\right)+\delta G_{N-1}^{i}\left(X_{N-1}\right)+\delta U_{N-2}^{i},
\end{aligned}
$$

where

$$
\begin{gathered}
\left(\begin{array}{c}
V_{1}^{r} \\
V_{2}^{r} \\
\vdots \\
V_{N-1}^{r}
\end{array}\right)=\Theta\left(\begin{array}{c}
e_{1}^{r} \\
e_{2}^{r} \\
\vdots \\
e_{N-1}^{r}
\end{array}\right), \\
\left(\begin{array}{c}
V_{1}^{i} \\
V_{2}^{i} \\
\vdots \\
V_{N-1}^{i}
\end{array}\right)=\Theta\left(\begin{array}{c}
e_{1}^{i} \\
e_{2}^{i} \\
\vdots \\
e_{N-1}^{i}
\end{array}\right),
\end{gathered}
$$

and $\Theta$ is a real coefficient matrix. Then the error dynamical systems (8) can be represented as

$$
\begin{aligned}
& \dot{e}^{r}(t)=\Phi(t) e^{r}(t), \\
& \dot{e}^{i}(t)=\Phi(t) e^{i}(t),
\end{aligned}
$$

where $\Phi(t)=\Sigma+\Theta$. Obviously, there is no unique choice for such a matrix $\Theta$ to ensure that the error dynamical system (13) is asymptotically stable at the origin. In general, $\Theta$ is defined as a state-dependent real coefficient matrix. Then we can obtain the sufficient stability conditions of system (13) by transforming it into a stable system with antisymmetric structure. Before we give our main result, the following lemma should be introduced first.

Lemma 1 (see [41]). If $\mathcal{\varepsilon}:[0, \infty] \rightarrow \mathbb{R}$ satisfies $\lim _{t \rightarrow \infty} \int_{0}^{t} \varepsilon^{2}(t) d t<\infty, \dot{\varepsilon}(t)$ exists, and it is bounded, then $\lim _{t \rightarrow \infty} \varepsilon(t)=0$.

Theorem 2. Synchronization and antisynchronization of $\mathrm{N}$ coupled complex chaotic systems (5) will occur by using the control laws (11), if the state-dependent real coefficient matrix $\Phi(t)=\Phi_{1}(t)+\Phi_{2}$ satisfies the following assumptions:

$$
\begin{aligned}
\Phi_{1}^{T}(t) & =-\Phi_{1}(t), \\
\Phi_{2} & =\operatorname{diag}\left(-\varphi_{1},-\varphi_{2}, \ldots,-\varphi_{N-1}\right),
\end{aligned}
$$


where $\varphi_{k}=\operatorname{diag}\left(\varphi_{k 1}, \varphi_{k 2}, \ldots, \varphi_{k n}\right)(k=1,2, \ldots, N-1)$ and $\varphi_{k l}>0(l=1,2, \ldots, n)$.

Proof. Choose a positive definite Lyapunov candidate function as

$$
V(t)=\frac{1}{2}\left(e^{r}(t)\right)^{T} e^{r}(t)+\frac{1}{2}\left(e^{i}(t)\right)^{T} e^{i}(t) .
$$

Then the time derivative of $V(t)$ along the trajectory of the error system (13) is as follows:

$$
\begin{aligned}
\dot{V}(t)= & \frac{1}{2}\left(\left(e^{r}(t)\right)^{T}\left(\Phi^{T}(t)+\Phi(t)\right) e^{r}(t)\right) \\
& +\frac{1}{2}\left(\left(e^{i}(t)\right)^{T}\left(\Phi^{T}(t)+\Phi(t)\right) e^{i}(t)\right) .
\end{aligned}
$$

Substitution of (14) into (16) yields

$$
\begin{aligned}
& \dot{V}(t)=\frac{1}{2}\left[\left(e^{r}(t)\right)^{T}\left(\Phi_{2}^{T}+\Phi_{2}\right) e^{r}(t)\right] \\
& +\frac{1}{2}\left[\left(e^{i}(t)\right)^{T}\left(\Phi_{2}^{T}+\Phi_{2}\right) e^{i}(t)\right]=\left(e^{r}(t)\right)^{T} \Phi_{2} e^{r}(t) \\
& +\left(e^{i}(t)\right)^{T} \Phi_{2} e^{i}(t) \\
& =\left(\left(e_{1}^{r}(t)\right)^{T},\left(e_{2}^{r}(t)\right)^{T}, \ldots,\left(e_{N-1}^{r}(t)\right)^{T}\right) \\
& \left(\begin{array}{cccccc}
-\varphi_{1} & 0 & 0 & \cdots & 0 & 0 \\
0 & -\varphi_{2} & 0 & \cdots & 0 & 0 \\
\vdots & \vdots & \vdots & \ddots & \vdots & \vdots \\
0 & 0 & 0 & \cdots & 0 & -\varphi_{N-1}
\end{array}\right)\left(\begin{array}{c}
e_{1}^{r}(t) \\
e_{2}^{r}(t) \\
\vdots \\
e_{N-1}^{r}(t)
\end{array}\right) \\
& +\left(\left(e_{1}^{i}(t)\right)^{T},\left(e_{2}^{i}(t)\right)^{T}, \ldots,\left(e_{N-1}^{i}(t)\right)^{T}\right) \\
& \cdot\left(\begin{array}{cccccc}
-\varphi_{1} & 0 & 0 & \cdots & 0 & 0 \\
0 & -\varphi_{2} & 0 & \cdots & 0 & 0 \\
\vdots & \vdots & \vdots & \ddots & \vdots & \vdots \\
0 & 0 & 0 & \cdots & 0 & -\varphi_{N-1}
\end{array}\right)\left(\begin{array}{c}
e_{1}^{i}(t) \\
e_{2}^{i}(t) \\
\vdots \\
e_{N-1}^{i}(t)
\end{array}\right) \\
& =-\left(e_{1}^{r}(t)\right)^{T} \varphi_{1} e_{1}^{r}(t)-\left(e_{2}^{r}(t)\right)^{T} \varphi_{2} e_{2}^{r}(t)-\cdots \\
& -\left(e_{N-1}^{r}(t)\right)^{T} \varphi_{N-1} e_{N-1}^{r}(t)-\left(e_{1}^{i}(t)\right)^{T} \varphi_{1} e_{1}^{i}(t) \\
& -\left(e_{2}^{i}(t)\right)^{T} \varphi_{2} e_{2}^{i}(t)-\cdots-\left(e_{N-1}^{i}(t)\right)^{T} \varphi_{N-1} e_{N-1}^{i}(t) \\
& \leq-\varphi_{\min }\left[\left(e_{1}^{r}(t)\right)^{T} e_{1}^{r}(t)+\left(e_{2}^{r}(t)\right)^{T} e_{2}^{r}(t)+\cdots\right. \\
& +\left(e_{N-1}^{r}(t)\right)^{T} e_{N-1}^{r}(t)+\left(e_{1}^{i}(t)\right)^{T} e_{1}^{i}(t)+\cdots \\
& \left.+\left(e_{N-1}^{i}(t)\right)^{T} e_{N-1}^{i}(t)\right]=-\varphi_{\min }\|e(t)\|^{2},
\end{aligned}
$$

where $\varphi_{\text {min }}=\min _{1 \leq k \leq N-1,1 \leq l \leq n}\left\{\varphi_{k l}\right\}$. Thus taking the integral of both sides, we have

$$
\int_{0}^{t}\|e(t)\|^{2} d t \leq \frac{V(0)-V(t)}{\varphi_{\min }} \leq \frac{V(0)}{\varphi_{\min }}<\infty .
$$

As $\dot{e}^{r}(t)$ and $\dot{e}^{i}(t)$ exist and they are bounded, then

$$
\begin{aligned}
& \frac{d(\|e(t)\|)}{d t} \\
& =\frac{1}{2}\|e(t)\|^{-1}\left[2\left(\dot{e}^{r}(t)\right)^{T} e^{r}(t)+2\left(\dot{e}^{i}(t)\right)^{T} e^{i}(t)\right] \\
& =\frac{\left(\dot{e}^{r}(t)\right)^{T} e^{r}(t)+\left(\dot{e}^{i}(t)\right)^{T} e^{i}(t)}{\|e(t)\|} .
\end{aligned}
$$

Hence, we get that the derivative of $\|e(t)\|$ exists and it is bounded. According to Lemma 1, we have $\lim _{t \rightarrow \infty}\|e(t)\|=$ 0 . That implies that the closed-loop system (13) is globally asymptotically stable. Therefore, $N$-coupled complex chaotic systems (5) realize synchronization and antisynchronization. This completes the proof.

The following corollary can be easily obtained from Theorem 2.

Corollary 3. If the structures of general complex chaotic systems are identical, that is, $A_{k}=A_{l}=A$ and $G_{k}(\cdot)=G_{l}(\cdot)=$ $G(\cdot)$, then complex controllers $U_{k}=U_{k}^{r}+j U_{k}^{i}$ can be constructed as

$$
\begin{aligned}
& U_{1}=V_{1}-\left((1-\delta) Q_{2}-\left(\delta^{N}-\delta\right) Q_{1}\right) X_{1}-G\left(X_{2}\right) \\
& +\delta G\left(X_{1}\right) \\
& U_{2}=V_{2}-(\delta-1)\left(Q_{2}-Q_{3}\right) X_{2}-G\left(X_{3}\right)+\delta G\left(X_{2}\right) \\
& +\delta U_{1} \\
& U_{N-1}=V_{N-1}-(\delta-1)\left(Q_{N-1}-Q_{N}\right) X_{N-1}-G\left(X_{N}\right) \\
& +\delta G\left(X_{N-1}\right)+\delta U_{N-2} \text {, }
\end{aligned}
$$

where $V_{k}=V_{k}^{r}+j V_{k}^{i}(k=1,2, \ldots, N-1)$ are defined as in (12) and $\Theta$ is a real coefficient matrix. Similar to the aforementioned discussion, we can achieve synchronization and antisynchronization of $N$-coupled identical complex chaotic systems with ring connection based on antisymmetric structure.

As above-mentioned analysis, we easily know that the error dynamical systems (8) can be transformed into system (13) by utilizing complex control laws $U_{k}$. Moreover, the suitable real coefficient matrix $\Theta$ is chosen to translate system (13) into a stable system with an antisymmetric structure. It is known that the antisymmetric structure in Theorem 2 is the generalization of the tridiagonal structure [42, 43]. The error system constructed with the antisymmetric structure is more convenient than the one with tridiagonal structure when 
the original system has some zero elements at the tridiagonal position and nonzero elements at other positions. And we find that selecting the coefficient matrix plays an important role in achieving synchronization and antisynchronization of $\mathrm{N}$-coupled complex chaotic systems with ring connection.

\section{Synchronization and Antisynchronization of $N$-Coupled Complex PMSM Systems with Ring Connection}

In the following, we investigate synchronization and antisynchronization of $N$-coupled complex PMSM systems with ring connection. Now, 4-coupled complex PMSM systems with ring connection and designed controllers can be described as

$$
\begin{aligned}
\dot{x}_{11}= & a\left(x_{12}-x_{11}\right)+q_{11}\left(x_{41}-x_{11}\right), \\
\dot{x}_{12}= & b x_{11}-x_{12}-x_{11} x_{13}+q_{12}\left(x_{42}-x_{12}\right), \\
\dot{x}_{13}= & \frac{1}{2}\left(\bar{x}_{11} x_{12}+x_{11} \bar{x}_{12}\right)-x_{13}+q_{13}\left(x_{43}-x_{13}\right), \\
\dot{x}_{21}= & a\left(x_{22}-x_{21}\right)+q_{21}\left(x_{11}-x_{21}\right)+u_{11}, \\
\dot{x}_{22}= & b x_{21}-x_{22}-x_{21} x_{23}+q_{22}\left(x_{12}-x_{22}\right)+u_{12}, \\
\dot{x}_{23}= & \frac{1}{2}\left(\bar{x}_{21} x_{22}+x_{21} \bar{x}_{22}\right)-x_{23}+q_{23}\left(x_{13}-x_{23}\right) \\
& +u_{13}, \\
\dot{x}_{31}= & a\left(x_{32}-x_{31}\right)+q_{31}\left(x_{21}-x_{31}\right)+u_{21},
\end{aligned}
$$

$$
\begin{aligned}
\dot{x}_{32}= & b x_{31}-x_{32}-x_{31} x_{33}+q_{32}\left(x_{22}-x_{32}\right)+u_{22}, \\
\dot{x}_{33}= & \frac{1}{2}\left(\bar{x}_{31} x_{32}+x_{31} \bar{x}_{32}\right)-x_{33}+q_{33}\left(x_{23}-x_{33}\right) \\
& +u_{23}, \\
\dot{x}_{41}= & a\left(x_{42}-x_{41}\right)+q_{41}\left(x_{31}-x_{41}\right)+u_{31}, \\
\dot{x}_{42}= & b x_{41}-x_{42}-x_{41} x_{43}+q_{42}\left(x_{32}-x_{42}\right)+u_{32}, \\
\dot{x}_{43}= & \frac{1}{2}\left(\bar{x}_{41} x_{42}+x_{41} \bar{x}_{42}\right)-x_{43}+q_{43}\left(x_{33}-x_{43}\right) \\
& +u_{33},
\end{aligned}
$$

where

$$
\begin{gathered}
A_{k}=A=\left(\begin{array}{ccc}
-a & a & 0 \\
b & -1 & 0 \\
0 & 0 & -1
\end{array}\right) \\
G_{k}\left(X_{k}\right)=G\left(X_{k}\right)=\left(\begin{array}{c}
0 \\
-x_{k 1} x_{k 3} \\
\frac{1}{2}\left(\bar{x}_{k 1} x_{k 2}+x_{k 1} \bar{x}_{k 2}\right)
\end{array}\right),
\end{gathered}
$$

$Q_{k}=\operatorname{diag}\left(q_{k 1}, q_{k 2}, q_{k 3}\right)$ are the coupled matrices $(k=1,2$, $3,4)$, and $U_{k}=\left(u_{k 1}, u_{k 2}, u_{k 3}\right)^{T}(k=1,2,3)$ are the complex control inputs.

The synchronization error can be presented as $e_{k}=X_{k+1}-$ $\delta X_{k}(k=1,2,3)$. Then the error dynamical system can be written in the form

$$
\begin{aligned}
\dot{e}(t)= & \left(\begin{array}{ccc}
A-\delta^{3} Q_{1}-Q_{2} & -\delta^{2} Q_{1} & -\delta Q_{1} \\
Q_{2} & A-Q_{3} & 0 \\
0 & Q_{3} & A-Q_{4}
\end{array}\right)\left(\begin{array}{c}
e_{1}(t) \\
e_{2}(t) \\
e_{3}(t)
\end{array}\right) \\
& +\left(\begin{array}{c}
\left((1-\delta) Q_{2}-\left(\delta^{4}-\delta\right) Q_{1}\right) X_{1}+G\left(X_{2}\right)-\delta G\left(X_{1}\right)+U_{1} \\
(\delta-1)\left(Q_{2}-Q_{3}\right) X_{2}+G\left(X_{3}\right)-\delta G\left(X_{2}\right)+U_{2}-\delta U_{1} \\
(\delta-1)\left(Q_{3}-Q_{4}\right) X_{3}+G\left(X_{4}\right)-\delta G\left(X_{3}\right)+U_{3}-\delta U_{2}
\end{array}\right) .
\end{aligned}
$$

4.1. Synchronization of 4-Coupled Complex PMSM Systems. When $\delta=1$, the error system is degraded into the following complete synchronization error system:

$\dot{e}^{r}(t)$

$$
\begin{aligned}
= & \left(\begin{array}{ccc}
A-Q_{1}-Q_{2} & -Q_{1} & -Q_{1} \\
Q_{2} & A-Q_{3} & 0 \\
0 & Q_{3} & A-Q_{4}
\end{array}\right)\left(\begin{array}{l}
e_{1}^{r}(t) \\
e_{2}^{r}(t) \\
e_{3}^{r}(t)
\end{array}\right) \\
& +\left(\begin{array}{c}
G^{r}\left(X_{2}\right)-G^{r}\left(X_{1}\right)+U_{1}^{r} \\
G^{r}\left(X_{3}\right)-G^{r}\left(X_{2}\right)+U_{2}^{r}-U_{1}^{r} \\
G^{r}\left(X_{4}\right)-G^{r}\left(X_{3}\right)+U_{3}^{r}-U_{2}^{r}
\end{array}\right),
\end{aligned}
$$

$\dot{e}^{i}(t)$

$$
\begin{gathered}
=\left(\begin{array}{ccc}
A-Q_{1}-Q_{2} & -Q_{1} & -Q_{1} \\
Q_{2} & A-Q_{3} & 0 \\
0 & Q_{3} & A-Q_{4}
\end{array}\right)\left(\begin{array}{c}
e_{1}^{i}(t) \\
e_{2}^{i}(t) \\
e_{3}^{i}(t)
\end{array}\right) \\
+\left(\begin{array}{c}
G^{i}\left(X_{2}\right)-G^{i}\left(X_{1}\right)+U_{1}^{i} \\
G^{i}\left(X_{3}\right)-G^{i}\left(X_{2}\right)+U_{2}^{i}-U_{1}^{i} \\
G^{i}\left(X_{4}\right)-G^{i}\left(X_{3}\right)+U_{3}^{i}-U_{2}^{i}
\end{array}\right) .
\end{gathered}
$$


Choose the real coefficient matrix $\Theta=\left(\begin{array}{c}\Theta_{1} \\ \Theta_{2} \\ \Theta_{3}\end{array}\right)$ as follows:

$$
\begin{aligned}
& \Theta_{1}=\left(\begin{array}{ccccccccc}
0 & 0 & 0 & 0 & x_{11}^{r} & 0 & 0 & 0 & 0 \\
-a-b & 0 & 0 & 0 & 0 & 0 & 0 & 0 & 0 \\
0 & 0 & 0 & 0 & 0 & 0 & 0 & 0 & 0
\end{array}\right), \\
& \Theta_{2}=\left(\begin{array}{ccccccccc}
q_{11}-q_{21} & 0 & 0 & 0 & 0 & 0 & -q_{31} & 0 & 0 \\
-x_{11}^{r} & q_{12}-q_{22} & 0 & -a-b & 0 & 0 & 0 & -q_{32} & 0 \\
0 & 0 & q_{13}-q_{23} & 0 & 0 & 0 & 0 & 0 & -q_{33}
\end{array}\right), \\
& \Theta_{3}=\left(\begin{array}{ccccccccc}
q_{11} & 0 & 0 & 0 & 0 & 0 & 0 & 0 & 0 \\
0 & q_{12} & 0 & 0 & 0 & 0 & -a-b & 0 & 0 \\
0 & 0 & q_{13} & 0 & 0 & 0 & 0 & 0 & 0
\end{array}\right)
\end{aligned}
$$

Thus, complex controllers $U_{k}(k=1,2,3)$ are provided as

$$
\begin{aligned}
U_{1}= & U_{1}^{r}+j U_{1}^{i}=\left(\begin{array}{c}
x_{11}^{r} e_{22}^{r} \\
-(a+b) e_{11}^{r}+x_{21}^{r} x_{23}-x_{11}^{r} x_{13} \\
-x_{21}^{r} x_{22}^{r}-x_{21}^{i} x_{22}^{i}+x_{11}^{r} x_{12}^{r}+x_{11}^{i} x_{12}^{i}
\end{array}\right)+j\left(\begin{array}{c}
x_{11}^{r} e_{22}^{i} \\
-(a+b) e_{11}^{i}+x_{21}^{i} x_{23}-x_{11}^{i} x_{13}
\end{array}\right), \\
U_{2}= & U_{2}^{r}+j U_{2}^{i} \\
= & \left(\begin{array}{c}
\left(q_{12}-q_{22}\right) e_{12}^{r}-(a+b)\left(e_{21}^{r}+e_{11}^{r}\right)-q_{32} e_{32}^{r}-x_{11}^{r} e_{11}^{r}+x_{31}^{r} x_{33}-x_{11}^{r} x_{13} \\
\left(q_{13}-q_{23}\right) e_{13}-q_{33} e_{33}-x_{31}^{r} x_{32}^{r}-x_{31}^{i} x_{32}^{i}+x_{11}^{r} x_{12}^{r}+x_{11}^{i} x_{12}^{i} \\
\left(q_{11}-q_{21}\right) e_{11}^{i}-q_{31} e_{31}^{i}+x_{11}^{r} e_{22}^{i}
\end{array}\right) \\
& +j\left(\begin{array}{c}
\left(q_{12}-q_{22}\right) e_{12}^{i}-(a+b)\left(e_{21}^{i}+e_{11}^{i}\right)-q_{32} e_{32}^{i}-x_{11}^{r} e_{11}^{i}+x_{31}^{i} x_{33}-x_{11}^{i} x_{13} \\
0
\end{array}\right)
\end{aligned}
$$

$$
\begin{aligned}
U_{3}= & U_{3}^{r}+j U_{3}^{i} \\
= & \left(\begin{array}{c}
\left(2 q_{11}-q_{21}\right) e_{11}^{r}-q_{31} e_{31}^{r}+x_{11}^{r} e_{22}^{r} \\
\left(2 q_{12}-q_{22}\right) e_{12}^{r}-(a+b)\left(e_{11}^{r}+e_{21}^{r}+e_{31}^{r}\right)-q_{32} e_{32}^{r}-x_{11}^{r} e_{11}^{r}+x_{41}^{r} x_{43}-x_{11}^{r} x_{13} \\
\left(2 q_{13}-q_{23}\right) e_{13}-q_{33} e_{33}-x_{41}^{r} x_{42}^{r}-x_{41}^{i} x_{42}^{i}+x_{11}^{r} x_{12}^{r}+x_{11}^{i} x_{12}^{i}
\end{array}\right) \\
& +j\left(\begin{array}{c}
\left(2 q_{11}-q_{21}\right) e_{11}^{i}-q_{31} e_{31}^{i}+x_{11}^{r} e_{22}^{i} \\
\left(2 q_{12}-q_{22}\right) e_{12}^{i}-(a+b)\left(e_{11}^{i}+e_{21}^{i}+e_{31}^{i}\right)-q_{32} e_{32}^{i}-x_{11}^{r} e_{11}^{i}+x_{41}^{i} x_{43}-x_{11}^{i} x_{13} \\
0
\end{array}\right) .
\end{aligned}
$$


So we have $\dot{e}^{r}(t)=\left(\Phi_{1}(t)+\Phi_{2}\right) e^{r}(t)$ and $\dot{e}^{i}(t)=\left(\Phi_{1}(t)+\right.$ $\left.\Phi_{2}\right) e^{i}(t)$, where $\Phi_{2}=\operatorname{diag}\left(-a-q_{11}-q_{21},-1-q_{12}-q_{22}\right.$, $-1-q_{13}-q_{23},-a-q_{31},-1-q_{32},-1-q_{33},-a-q_{41},-1-q_{42}$, $\left.-1-q_{43}\right)$ and

$$
\begin{gathered}
\Phi_{1}(t) \\
=\left(\begin{array}{ccccccccc}
0 & a & 0 & -q_{11} & x_{11}^{r} & 0 & -q_{11} & 0 & 0 \\
-a & 0 & 0 & 0 & -q_{12} & 0 & 0 & -q_{12} & 0 \\
0 & 0 & 0 & 0 & 0 & -q_{13} & 0 & 0 & -q_{13} \\
q_{11} & 0 & 0 & 0 & a & 0 & -q_{31} & 0 & 0 \\
-x_{11}^{r} & q_{12} & 0 & -a & 0 & 0 & 0 & -q_{32} & 0 \\
0 & 0 & q_{13} & 0 & 0 & 0 & 0 & 0 & -q_{33} \\
q_{11} & 0 & 0 & q_{31} & 0 & 0 & 0 & a & 0 \\
0 & q_{12} & 0 & 0 & q_{32} & 0 & -a & 0 & 0 \\
0 & 0 & q_{13} & 0 & 0 & q_{33} & 0 & 0 & 0
\end{array}\right) .
\end{gathered}
$$

If the conditions

$$
\begin{array}{r}
-a-q_{11}-q_{21}<0, \\
-1-q_{12}-q_{22}<0, \\
-1-q_{13}-q_{23}<0, \\
-a-q_{31}<0, \\
-1-q_{32}<0, \\
-1-q_{33}<0, \\
-a-q_{41}<0,
\end{array}
$$

$$
\begin{aligned}
& \Theta_{1}=\left(\begin{array}{ccccccccc}
0 & -a-b & 0 & q_{11}-q_{21} & 0 & 0 & -q_{11} & 0 & 0 \\
0 & 0 & 0 & 0 & q_{12}-q_{22} & 0 & 0 & -q_{12} & 0 \\
0 & 0 & 0 & 0 & 0 & q_{13}-q_{23} & 0 & 0 & -q_{13}
\end{array}\right), \\
& \Theta_{2}=\left(\begin{array}{ccccccccc}
0 & 0 & 0 & 0 & -a-b & 0 & -q_{31} & 0 & 0 \\
0 & 0 & 0 & 0 & 0 & 0 & 0 & -q_{32} & 0 \\
0 & 0 & 0 & 0 & 0 & 0 & 0 & 0 & -q_{33}
\end{array}\right) \\
& \Theta_{3}=\left(\begin{array}{ccccccccc}
0 & 0 & 0 & 0 & 0 & 0 & 0 & -a-b-x_{32}^{r} & 0 \\
0 & 0 & 0 & 0 & 0 & 0 & x_{32}^{r} & 0 & 0 \\
0 & 0 & 0 & 0 & 0 & 0 & 0 & 0 & 0
\end{array}\right),
\end{aligned}
$$

$$
\begin{aligned}
& -1-q_{42}<0 \\
& -1-q_{43}<0
\end{aligned}
$$

hold, then the error system is asymptotically stable due to Theorem 2. Hence, complete synchronization of 4-coupled complex PMSM systems is realized.

The simulation results are illustrated with the initial condition $X_{1}(0)=(1+2 j, 3+4 j, 5)^{T}, X_{2}(0)=(6+16 j, 15+$ $14 j, 30)^{T}, X_{3}(0)=(10+4 j, 10+21 j, 8)^{T}$, and $X_{4}(0)=$ $(5+9 j, 11+4 j, 10)^{T}$. Furthermore, selecting $q_{k 1}=q_{k 2}=$ $q_{k 3}=1(k=1,2,3,4)$, we obtain simulation results as shown in Figures 3 and 4 . Figure 3 displays the state variables for complete synchronization of the 4-coupled complex PMSM systems. As we can see from Figure 4 the errors of synchronization converge asymptotically to zero in a quite short period. Thus, we achieve complete synchronization of 4-coupled complex PMSM systems with ring connection.

4.2. Antisynchronization of 4-Coupled Complex PMSM Systems. When $\delta=-1$, the error system is degraded into the following antisynchronization error system:

$$
\begin{aligned}
\dot{e}(t) & =\left(\begin{array}{ccc}
A+Q_{1}-Q_{2} & -Q_{1} & Q_{1} \\
Q_{2} & A-Q_{3} & 0 \\
0 & Q_{3} & A-Q_{4}
\end{array}\right)\left(\begin{array}{c}
e_{1}(t) \\
e_{2}(t) \\
e_{3}(t)
\end{array}\right) \\
& +\left(\begin{array}{c}
2\left(Q_{2}-Q_{1}\right) X_{1}+G\left(X_{2}\right)+G\left(X_{1}\right)+U_{1} \\
2\left(Q_{3}-Q_{2}\right) X_{2}+G\left(X_{3}\right)+G\left(X_{2}\right)+U_{2}+U_{1} \\
2\left(Q_{4}-Q_{3}\right) X_{3}+G\left(X_{4}\right)+G\left(X_{3}\right)+U_{3}+U_{2}
\end{array}\right) .
\end{aligned}
$$

By choosing the real coefficient matrix $\Theta=\left(\begin{array}{c}\Theta_{1} \\ \Theta_{2} \\ \Theta_{3}\end{array}\right)$, we design the complex controllers $U_{k}=\left(u_{k 1}, u_{k 2}, u_{k 3}\right)^{T}(k=$ $1,2,3)$ as

$$
\begin{aligned}
u_{11}= & -(a+b) e_{12}+\left(q_{11}-q_{21}\right) e_{21}-q_{11} e_{31} \\
& -2\left(q_{21}-q_{11}\right) x_{11},
\end{aligned}
$$



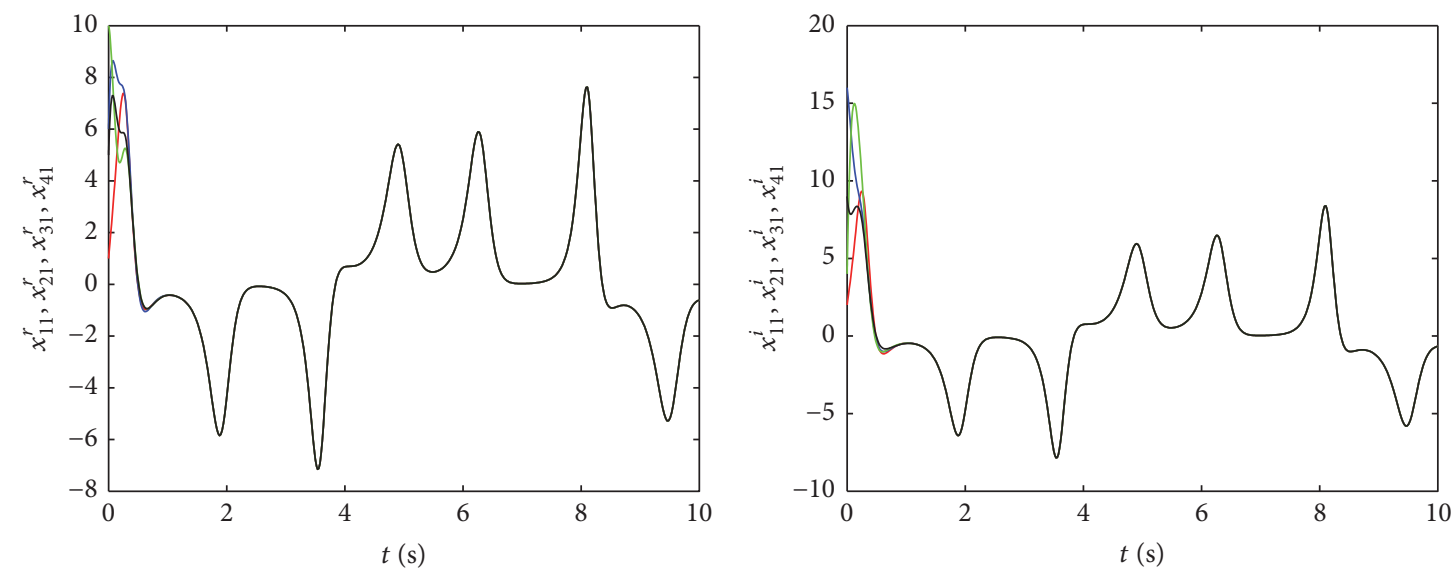

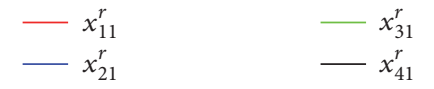

(a)

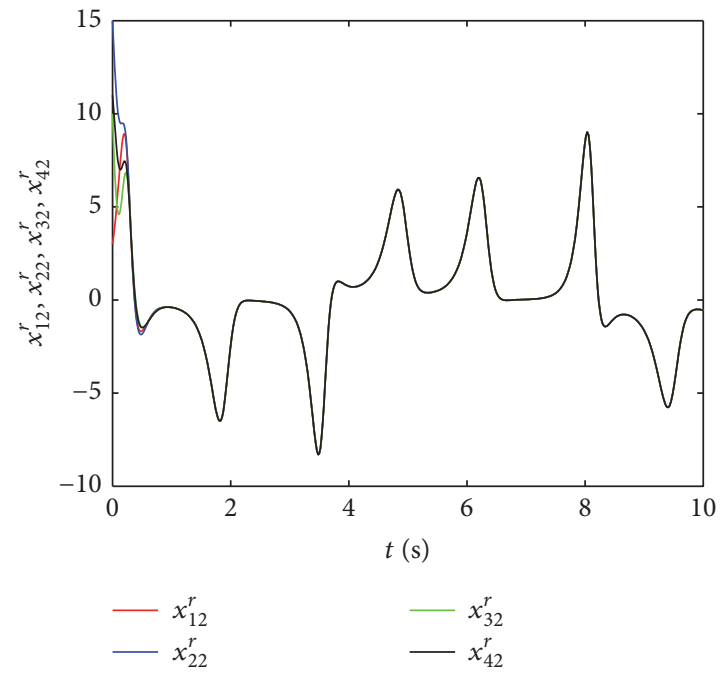

(c)

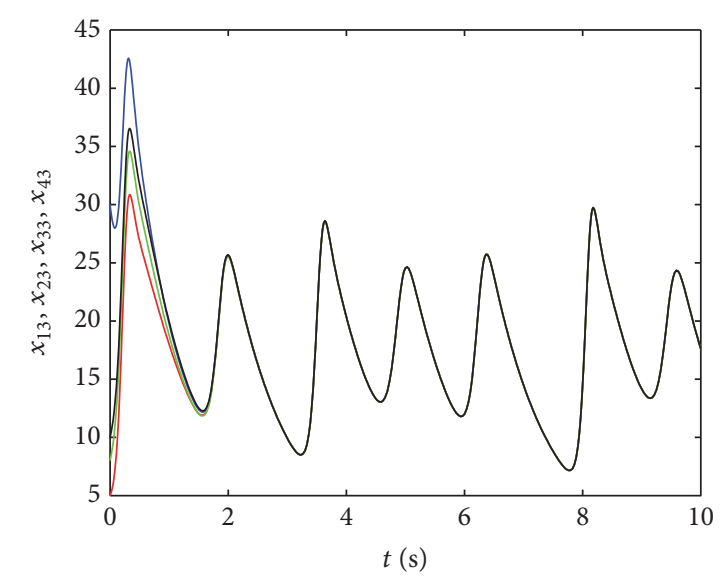

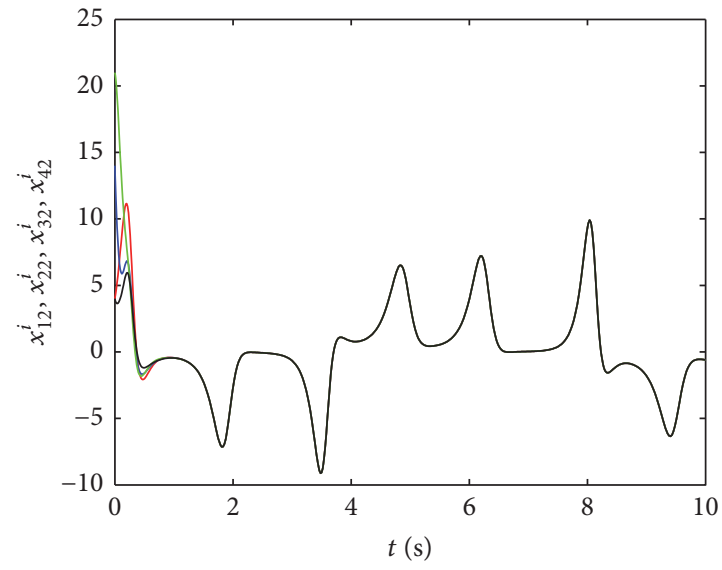

$\begin{array}{ll}-x_{12}^{i} & -x_{32}^{i} \\ -x_{22}^{i} & -x_{42}^{i}\end{array}$

(d)

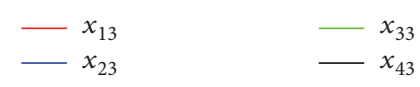

(e)

FIGURE 3: The state trajectories $x_{k 1}, x_{k 2}$, and $x_{k 3}(k=1,2,3,4)$ for synchronization of 4 -coupled complex PMSM systems. 

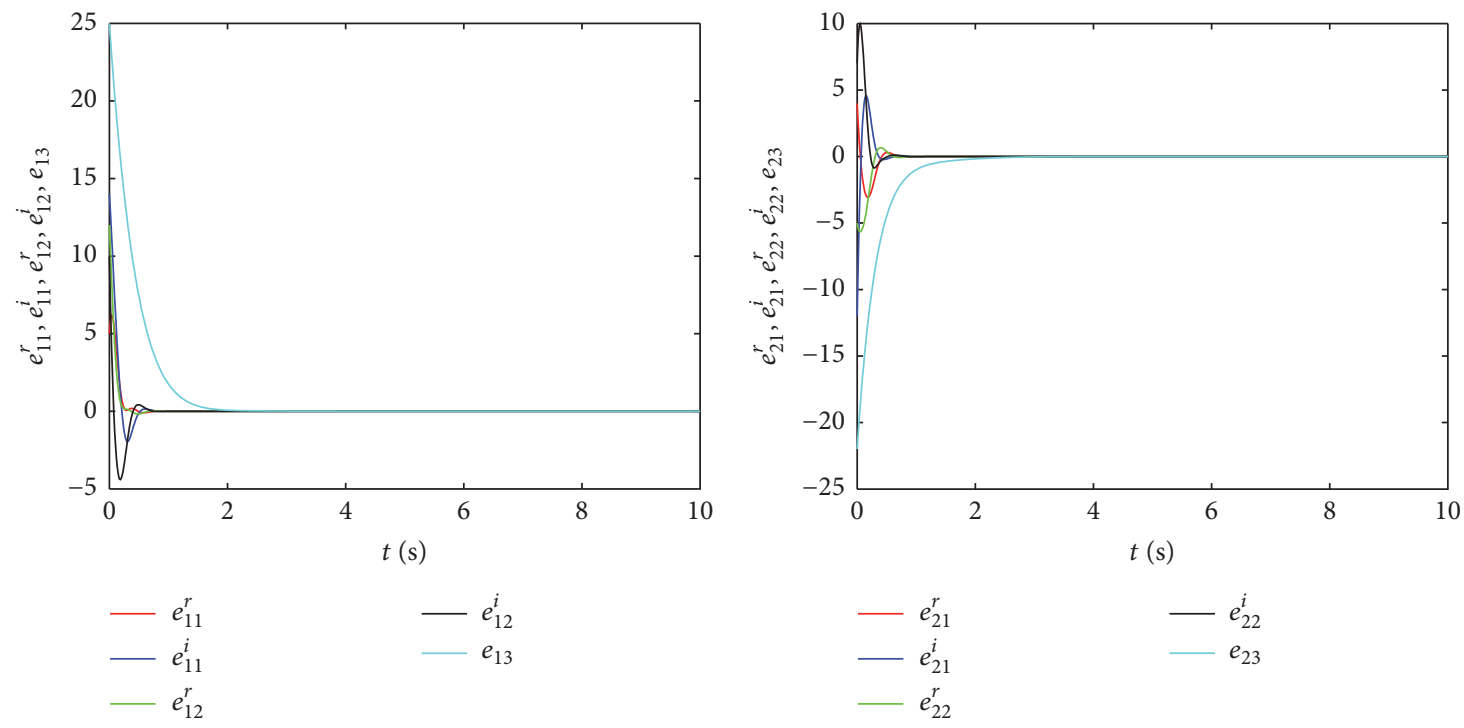

(a)

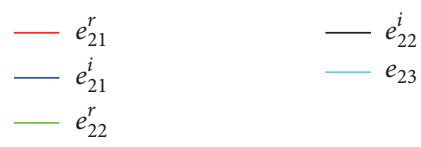

(b)

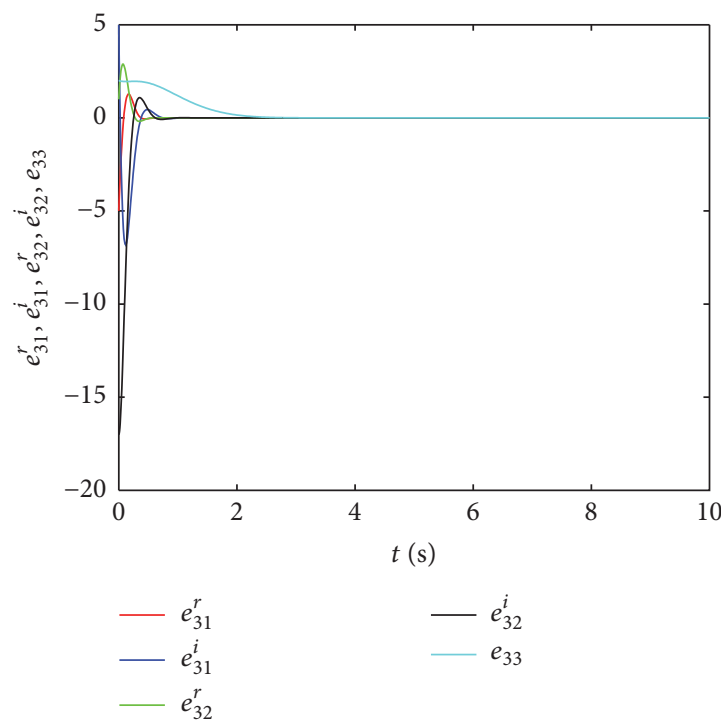

(c)

FIGURE 4: The error dynamics of synchronization among 4-coupled complex PMSM systems.

$$
\begin{aligned}
u_{21}= & (a+b)\left(e_{12}-e_{22}\right)+\left(q_{11}-q_{31}\right) e_{31} \\
& -\left(q_{11}-q_{21}\right)\left(e_{21}+2 x_{11}\right) \\
& +2\left(q_{21}-q_{31}\right) x_{21}, \\
u_{22}= & \left(q_{12}-q_{32}\right) e_{32}-\left(q_{12}-q_{22}\right)\left(e_{22}+2 x_{12}\right) \\
& +2\left(q_{22}-q_{32}\right) x_{22}+x_{31} x_{33}-x_{11} x_{13}, \\
u_{23}= & \left(q_{13}-q_{33}\right) e_{33}-\left(q_{13}-q_{23}\right)\left(e_{23}+2 x_{13}\right) \\
& +2\left(q_{23}-q_{33}\right) x_{23}-x_{31}^{r} x_{32}^{r}-x_{31}^{i} x_{32}^{i} \\
& +x_{11}^{r} x_{12}^{r}+x_{11}^{i} x_{12}^{i}, \\
= & (a+b)\left(e_{22}-e_{32}-e_{12}\right)+\left(q_{31}-q_{11}\right) e_{31}
\end{aligned}
$$

$$
\begin{aligned}
& -x_{32}^{r} e_{32}+\left(q_{11}-q_{21}\right)\left(e_{21}+2 x_{11}\right) \\
& +2\left(q_{31}-q_{41}\right) x_{31}-2\left(q_{21}-q_{31}\right) x_{21}, \\
u_{32}= & \left(q_{32}-q_{12}\right) e_{32}+\left(q_{12}-q_{22}\right)\left(e_{22}+2 x_{12}\right) \\
& +x_{32}^{r} e_{31}+2\left(q_{32}-q_{42}\right) x_{32} \\
& -2\left(q_{22}-q_{32}\right) x_{22}+x_{41} x_{43}+x_{11} x_{13}, \\
u_{33}= & \left(q_{33}-q_{13}\right) e_{33}+\left(q_{13}-q_{23}\right)\left(e_{23}+2 x_{13}\right) \\
& +2\left(q_{33}-q_{43}\right) x_{33}-2\left(q_{23}-q_{33}\right) x_{23} \\
& -x_{41}^{r} x_{42}^{r}-x_{41}^{i} x_{42}^{i}-x_{11}^{r} x_{12}^{r}-x_{11}^{i} x_{12}^{i} .
\end{aligned}
$$



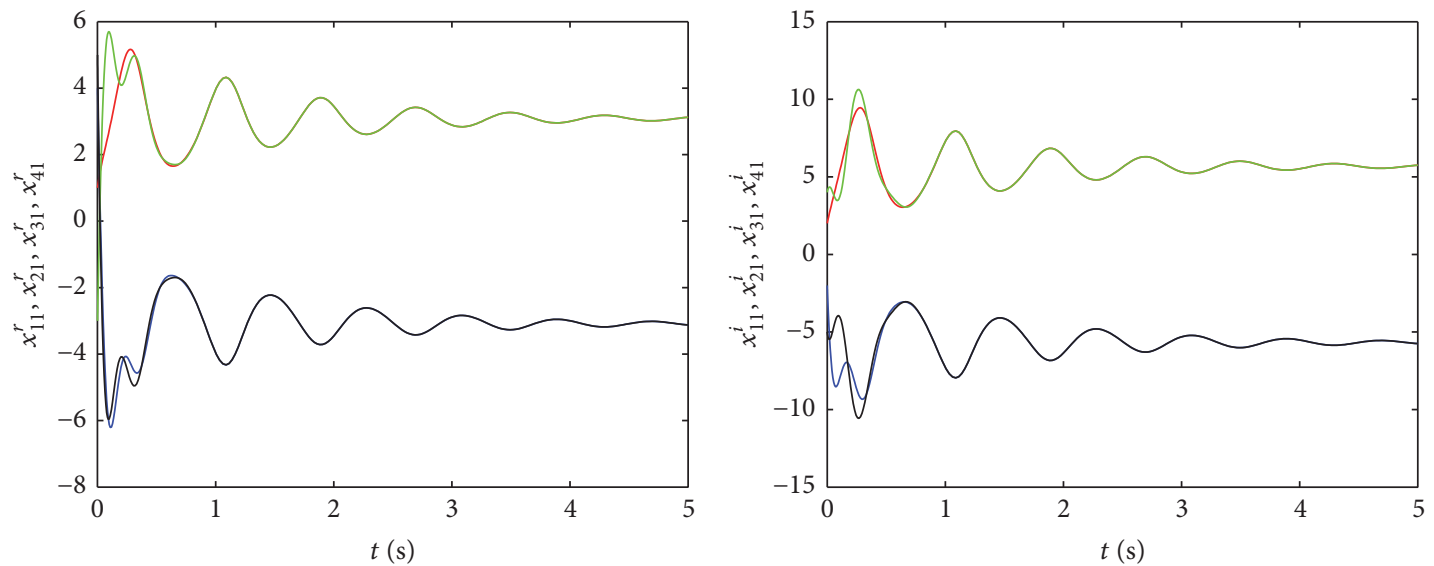

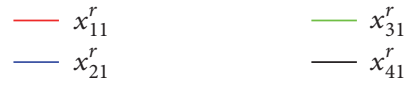

(a)

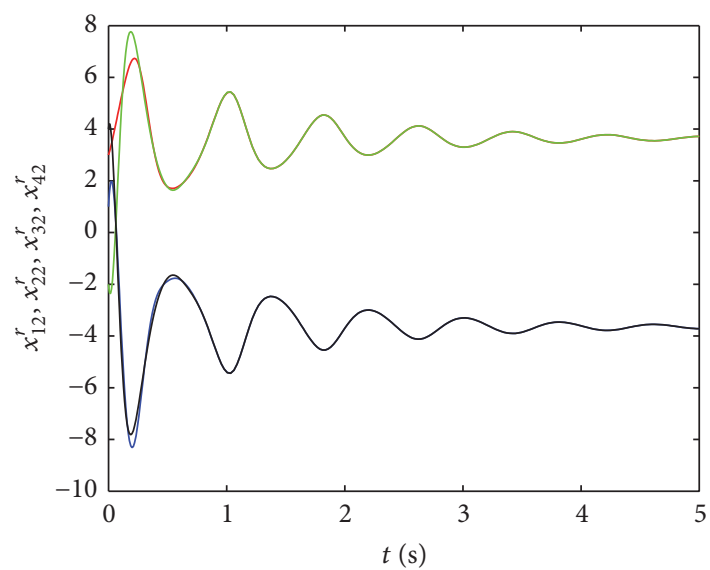

$\begin{array}{ll}-x_{12}^{r} & -x_{32}^{r} \\ -x_{22}^{r} & x_{42}^{r}\end{array}$

(c)

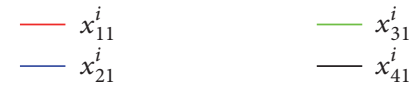

(b)

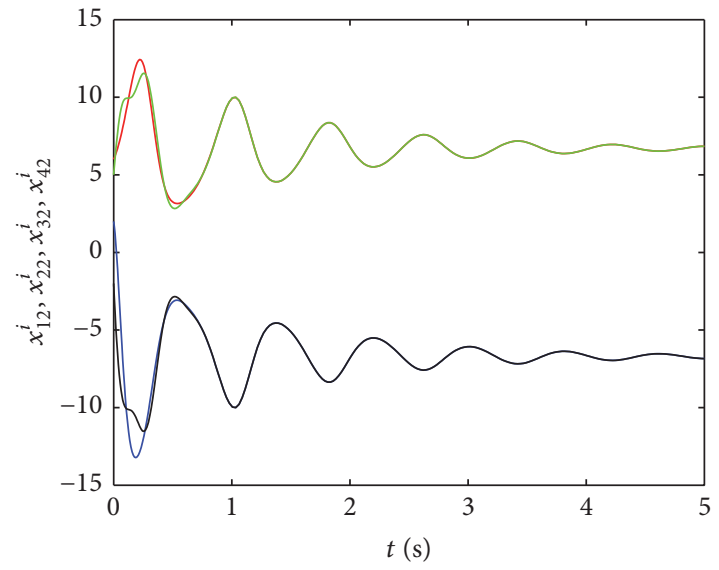

$-x_{12}^{i}$

$x_{32}^{i}$
$-x_{42}^{i}$

(d)

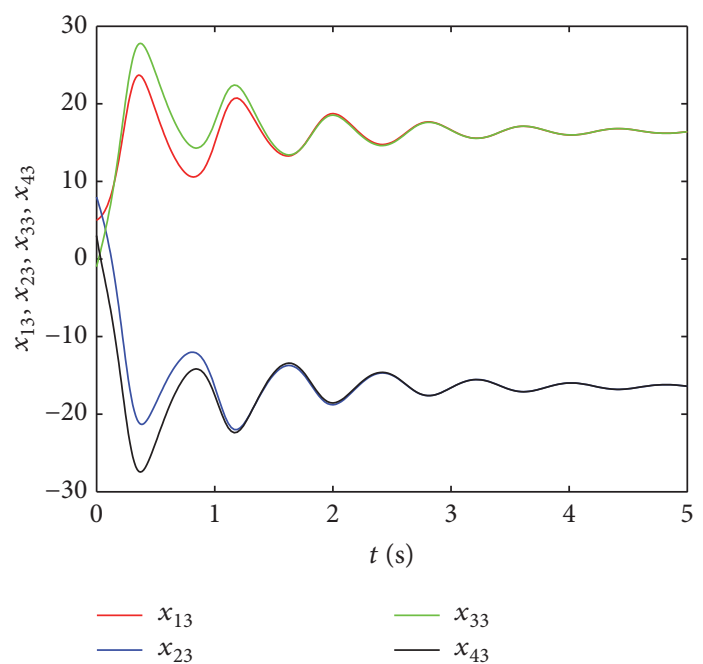

(e)

FIGURE 5: The state trajectories $x_{k 1}, x_{k 2}$, and $x_{k 3}(k=1,2,3,4)$ for antisynchronization of 4-coupled complex PMSM systems. 


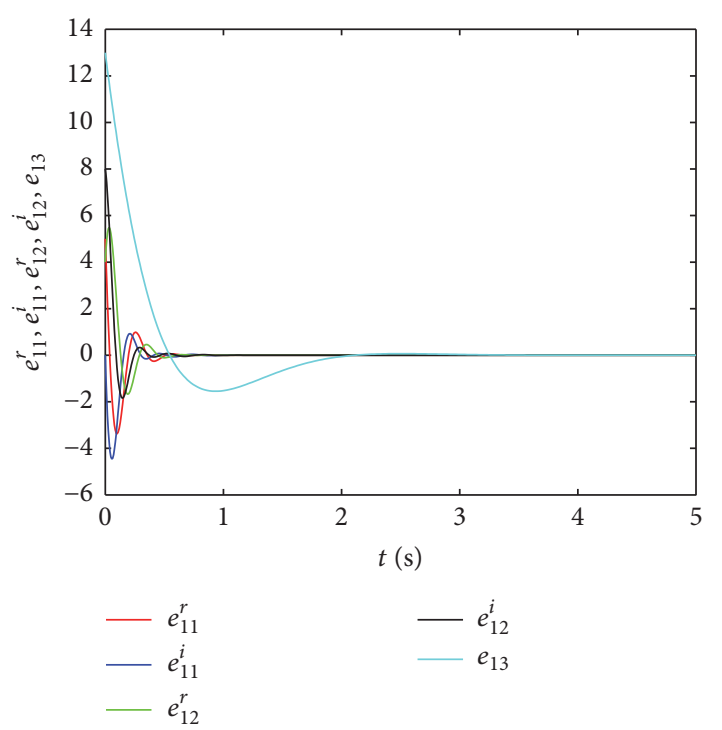

(a)
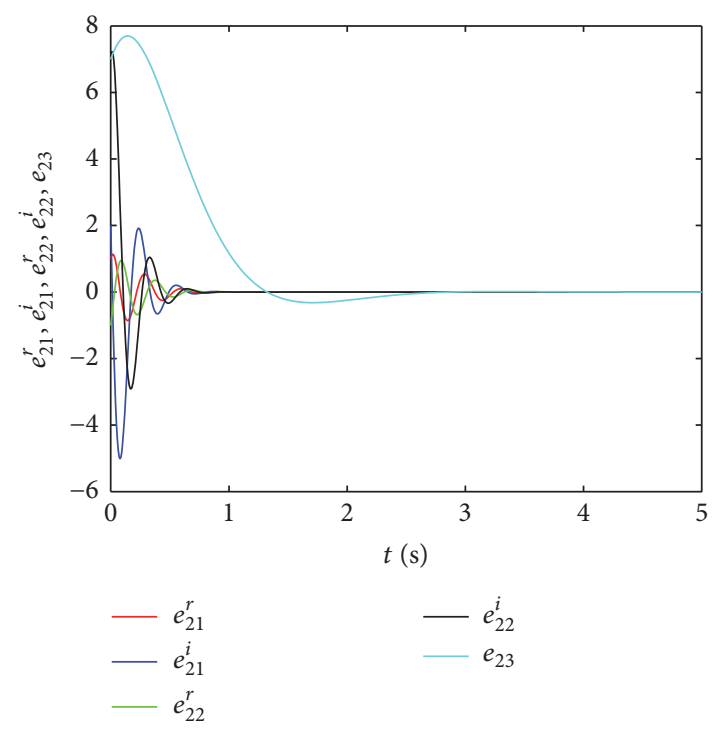

(b)

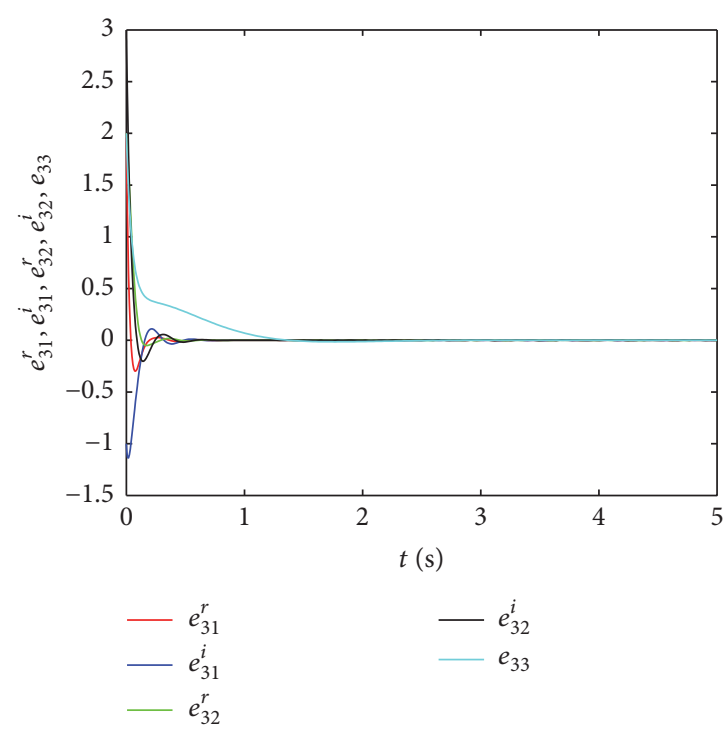

(c)

FIGURE 6: The error dynamics of antisynchronization among 4-coupled complex PMSM systems.

Then, we obtain the following error system $\dot{e}(t)=\Phi(t) e(t)=-1+q_{13}-q_{23},-a-q_{31},-1-q_{32},-1-q_{33},-a-q_{41},-1-q_{42}$, $\left(\Phi_{1}(t)+\Phi_{2}\right) e(t)$, where $\Phi_{2}=\operatorname{diag}\left(-a+q_{11}-q_{21},-1+q_{12}-q_{22}, \quad-1-q_{43}\right)$ and

$$
\Phi_{1}(t)=\left(\begin{array}{ccccccccc}
0 & -b & 0 & -q_{21} & 0 & 0 & 0 & 0 & 0 \\
b & 0 & 0 & 0 & -q_{22} & 0 & 0 & 0 & 0 \\
0 & 0 & 0 & 0 & 0 & -q_{23} & 0 & 0 & 0 \\
q_{21} & 0 & 0 & 0 & -b & 0 & -q_{31} & 0 & 0 \\
0 & q_{22} & 0 & b & 0 & 0 & 0 & -q_{32} & 0 \\
0 & 0 & q_{23} & 0 & 0 & 0 & 0 & 0 & -q_{33} \\
0 & 0 & 0 & q_{31} & 0 & 0 & 0 & -b-x_{32}^{r} & 0 \\
0 & 0 & 0 & 0 & q_{32} & 0 & b+x_{32}^{r} & 0 & 0 \\
0 & 0 & 0 & 0 & 0 & q_{33} & 0 & 0 & 0
\end{array}\right) .
$$


If the conditions

$$
\begin{array}{r}
-a+q_{11}-q_{21}<0, \\
-1+q_{12}-q_{22}<0, \\
-1+q_{13}-q_{23}<0, \\
-a-q_{31}<0, \\
-1-q_{32}<0, \\
-1-q_{33}<0, \\
-a-q_{41}<0, \\
-1-q_{42}<0, \\
-1-q_{43}<0
\end{array}
$$

hold, then the error system is asymptotically stable under complex controllers $U_{k}(k=1,2,3)$ according to Theorem 2 . Hence, antisynchronization of 4-coupled complex PMSM systems is realized.

The simulation results are illustrated with the initial condition $X_{1}(0)=(1+2 j, 3+6 j, 5)^{T}, X_{2}(0)=(4-2 j, 1+$ $2 j, 8)^{T}, X_{3}(0)=(-3+4 j,-2+5 j,-1)^{T}$, and $X_{4}(0)=$ $(5-5 j, 4-2 j, 3)^{T}$. Furthermore, selecting $q_{11}=q_{12}=$ $q_{13}=q_{31}=q_{32}=q_{33}=1, q_{21}=q_{22}=q_{23}=2$, and $q_{41}=q_{42}=q_{43}=20$, we have simulation results as displayed in Figures 5 and 6. Figure 5 shows the state variables for antisynchronization of the 4-coupled complex PMSM systems. It follows from Figure 6 that the errors of synchronization converge asymptotically to zero in a quite short period. As expected, antisynchronization of 4-coupled complex PMSM systems can occur.

\section{Conclusions}

A lot of progress has been made in the research of dynamical properties and synchronization for PMSM system with real variables, while the study on complex PMSM system is seldom reported in the previous literatures. So this paper analyzes and validates synchronization and antisynchronization of $N$-coupled complex PMSM systems with ring connection. First of all, for $N$-coupled general complex chaotic systems described by a unified mathematical expression with ring connection, we realize synchronization and antisynchronization based on the direct design method which is flexible and is suitable both for design and for implementation in practice. Thus, the proposed method is successfully applied to achieve synchronization and antisynchronization of $N$ coupled complex PMSM systems with ring connection on the basis of antisymmetric structure. Moreover, the synchronization scheme is so universal and simple that can be applicable to all complex chaotic systems, including those that can exhibit hyperchaotic behavior. Therefore, it is believed that the proposed scheme will play an important role in engineering practice.

\section{Competing Interests}

The authors declare that they have no competing interests.

\section{Acknowledgments}

The work is supported by Key Program of National Natural Science Foundation of China (no. 61533011) and the National Natural Science Foundation of China (no. 61273088, 61603203).

\section{References}

[1] A. C. Fowler, J. D. Gibbon, and M. J. McGuinness, “The complex Lorenz equations," Physica D, vol. 4, no. 2, pp. 139-163, 1982.

[2] E. Roldán, G. J. de Valcárcel, R. Vilaseca, and P. Mandel, "Singlemode-laser phase dynamics," Physical Review A, vol. 48, no. 1, pp. 591-598, 1993.

[3] C.-Z. Ning and H. Haken, "Detuned lasers and the complex Lorenz equations: Subcritical and supercritical Hopf bifurcations," Physical Review A, vol. 41, no. 7, pp. 3826-3837, 1990.

[4] V. Y. Toronov and V. L. Derbov, "Boundedness of attractors in the complex Lorenz model," Physical Review E, vol. 55, no. 3, pp. 3689-3692, 1997.

[5] G. M. Mahmoud, E. E. Mahmoud, and A. A. Arafa, "On projective synchronization of hyperchaotic complex nonlinear systems based on passive theory for secure communications," Physica Scripta, vol. 87, no. 5, Article ID 055002, 2013.

[6] P. Liu, H. J. Song, and X. Li, "Observe-based projective synchronization of chaotic complex modified van der pol-duffing oscillator with application to secure communication," Journal of Computational and Nonlinear Dynamics, vol. 10, no. 5, Article ID 051015, 2015.

[7] S. T. Liu and F. F. Zhang, "Complex function projective synchronization of complex chaotic system and its applications in secure communication," Nonlinear Dynamics, vol. 76, no. 2, pp. 1087-1097, 2014.

[8] L. Wang, B. Yang, and A. Abraham, "Distilling middle-age cement hydration kinetics from observed data using phased hybrid evolution," Soft Computing, vol. 20, pp. 3637-3656, 2016.

[9] L. Wang, B. Yang, Y. Chen, X. Q. Zhang, and J. Orchard, "Improving neural-network classifiers using nearest neighbor partitioning," IEEE Transactions on Neural Networks and Learning Systems, 2016.

[10] G. M. Mahmoud and E. E. Mahmoud, "Complete synchronization of chaotic complex nonlinear systems with uncertain parameters," Nonlinear Dynamics, vol. 62, no. 4, pp. 875-882, 2010.

[11] S. Liu and P. Liu, "Adaptive anti-synchronization of chaotic complex nonlinear systems with unknown parameters," Nonlinear Analysis: Real World Applications, vol. 12, no. 6, pp. 30463055,2011

[12] P. Liu and S. T. Liu, "Anti-synchronization between different chaotic complex systems," Physica Scripta, vol. 83, no. 6, Article ID 065006, 2011.

[13] G. M. Mahmoud and E. E. Mahmoud, "Synchronization and control of hyperchaotic complex Lorenz system," Mathematics and Computers in Simulation, vol. 80, no. 12, pp. 2286-2296, 2010. 
[14] G. M. Mahmoud and E. E. Mahmoud, "Lag synchronization of hyperchaotic complex nonlinear systems," Nonlinear Dynamics, vol. 67 , no. 2, pp. 1613-1622, 2012.

[15] E. E. Mahmoud, "Complex complete synchronization of two nonidentical hyperchaotic complex nonlinear systems," Mathematical Methods in the Applied Sciences, vol. 37, no. 3, pp. 321328, 2014.

[16] Z. Y. Wu, J. Q. Duan, and X. C. Fu, "Complex projective synchronization in coupled chaotic complex dynamical systems," Nonlinear Dynamics, vol. 69, no. 3, pp. 771-779, 2012.

[17] F. Zhang and S. Liu, "Full state hybrid projective synchronization and parameters identification for uncertain chaotic (hyperchaotic) complex systems," Journal of Computational and Nonlinear Dynamics, vol. 9, no. 2, Article ID 021009, 2014.

[18] G. M. Mahmoud and E. E. Mahmoud, "Complex modified projective synchronization of two chaotic complex nonlinear systems," Nonlinear Dynamics, vol. 73, no. 4, pp. 2231-2240, 2013.

[19] X. B. Zhou, M. R. Jiang, and Y. Q. Huang, "Combination synchronization of three identical or different nonlinear complex hyperchaotic systems," Entropy, vol. 15, no. 9, pp. 3746-3761, 2013.

[20] X. Zhou, L. Xiong, and X. Cai, "Combination-combination synchronization of four nonlinear complex chaotic systems," Abstract and Applied Analysis, vol. 2014, Article ID 953265, 14 pages, 2014.

[21] J. Sun, G. Cui, Y. Wang, and Y. Shen, "Combination complex synchronization of three chaotic complex systems," Nonlinear Dynamics, vol. 79, no. 2, pp. 953-965, 2015.

[22] C. M. Jiang and S. T. Liu, "Generalized combination complex synchronization of new hyperchaotic complex Lü-like systems," Advances in Difference Equations, vol. 2015, article 214, 2015.

[23] S. B. Wang, X. Y. Wang, X. Y. Wang, and Y. F. Zhou, "Adaptive generalized combination complex synchronization of uncertain real and complex nonlinear systems," AIP Advances, vol. 6, no. 4, Article ID 045011, 2016.

[24] I. M. Kyprianidis and I. N. Stouboulos, "Chaotic synchronization of three coupled oscillators with ring connection," Chaos, Solitons \& Fractals, vol. 17, no. 2-3, pp. 327-336, 2003.

[25] Y. G. Yu and S. C. Zhang, "Global synchronization of three coupled chaotic systems with ring connection," Chaos, Solitons and Fractals, vol. 24, no. 5, pp. 1233-1242, 2005.

[26] J.-A. Lu, X.-P. Han, Y.-T. Li, and M.-H. Yu, "Adaptive coupled synchronization among multi-Lorenz systems family," Chaos, Solitons and Fractals, vol. 31, no. 4, pp. 866-878, 2007.

[27] X. Y. Chen, J. L. Qiu, Q. Song, and A. C. Zhang, "Synchronization of $N$ coupled chaotic systems with ring connection based on special antisymmetric structure," Abstract and Applied Analysis, vol. 2013, Article ID 680604, 7 pages, 2013.

[28] X. Y. Chen, C. Y. Wang, and J. L. Qiu, "Synchronization and antisynchronization of $N$ different coupled chaotic systems with ring connection," International Journal of Modern Physics C, vol. 25, no. 5, Article ID 1440011, 2014.

[29] X. Chen, J. Qiu, J. Cao, and H. He, "Hybrid synchronization behavior in an array of coupled chaotic systems with ring connection," Neurocomputing, vol. 173, pp. 1299-1309, 2016.

[30] X.-Y. Wang and J.-M. Song, "Synchronization of the fractional order hyperchaos Lorenz systems with activation feedback control," Communications in Nonlinear Science and Numerical Simulation, vol. 14, no. 8, pp. 3351-3357, 2009.
[31] X. Y. Wang and Y. J. He, "Projective synchronization of fractional order chaotic system based on linear separation," Physics Letters A, vol. 372, no. 4, pp. 435-441, 2008.

[32] D. Lin and X. Y. Wang, "Observer-based decentralized fuzzy neural sliding mode control for interconnected unknown chaotic systems via network structure adaptation," Fuzzy Sets and Systems, vol. 161, no. 15, pp. 2066-2080, 2010.

[33] N. Cai, Y. Jing, and S. Zhang, "Generalized projective synchronization of different chaotic systems based on antisymmetric structure," Chaos, Solitons and Fractals, vol. 42, no. 2, pp. 11901196, 2009.

[34] M. Karabacak and H. I. Eskikurt, "Speed and current regulation of a permanent magnet synchronous motor via nonlinear and adaptive backstepping control," Mathematical and Computer Modelling, vol. 53, no. 9-10, pp. 2015-2030, 2011.

[35] M. Zribi, A. Oteafy, and N. Smaoui, "Controlling chaos in the permanent magnet synchronous motor," Chaos, Solitons and Fractals, vol. 41, no. 3, pp. 1266-1276, 2009.

[36] Q. Wei and X.-Y. Wang, "Chaos controlling of permanent magnet synchronous motor base on dither signal," Journal of Vibration and Control, vol. 19, no. 16, pp. 2541-2550, 2013.

[37] Q. Wei, X.-Y. Wang, and X.-P. Hu, "Optimal control for permanent magnet synchronous motor," Journal of Vibration and Control, vol. 20, no. 8, pp. 1176-1184, 2014.

[38] Q. Wei, X.-Y. Wang, and X.-P. Hu, "Inverse optimal control for permanent magnet synchronous motor," Journal of Vibration and Control, vol. 21, no. 4, pp. 801-807, 2015.

[39] X.-Y. Wang and H. Zhang, "Backstepping-based lag synchronization of a complex permanent magnet synchronous motor system," Chinese Physics B, vol. 22, no. 4, Article ID 048902, 2013.

[40] F. C. Zhang, C. L. Mu, X. Y. Wang, I. Ahmed, and Y. L. Shu, "Solution bounds of a new complex PMSM system," Nonlinear Dynamics, vol. 74, no. 4, pp. 1041-1051, 2013.

[41] G. Tao, "A simple alternative to the Barbalat lemma," IEEE Transactions on Automatic Control, vol. 42, no. 5, 698 pages, 1997.

[42] B. Liu, Y. M. Zhou, M. Jiang, and Z. K. Zhang, "Synchronizing chaotic systems using control based on tridiagonal structure," Chaos, Solitons and Fractals, vol. 39, no. 5, pp. 2274-2281, 2009.

[43] B. Liu and Z. K. Zhang, "Stability of nonlinear systems with tridiagonal structure and its applications," Acta Automatica Sinica, vol. 33, no. 4, pp. 442-445, 2007. 


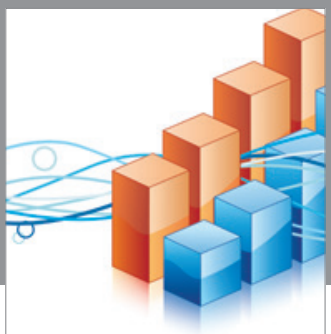

Advances in

Operations Research

vatem alat4

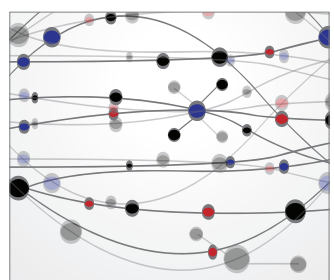

\section{The Scientific} World Journal
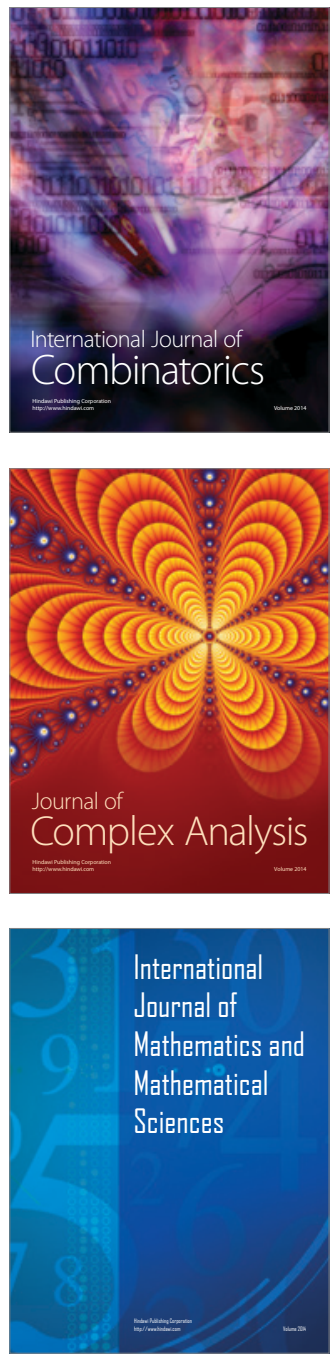
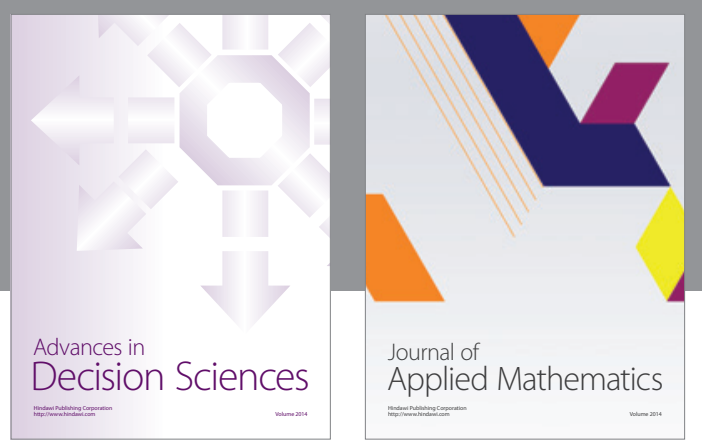

Algebra

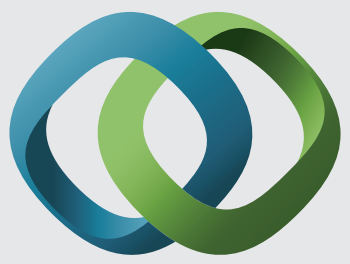

\section{Hindawi}

Submit your manuscripts at

https://www.hindawi.com
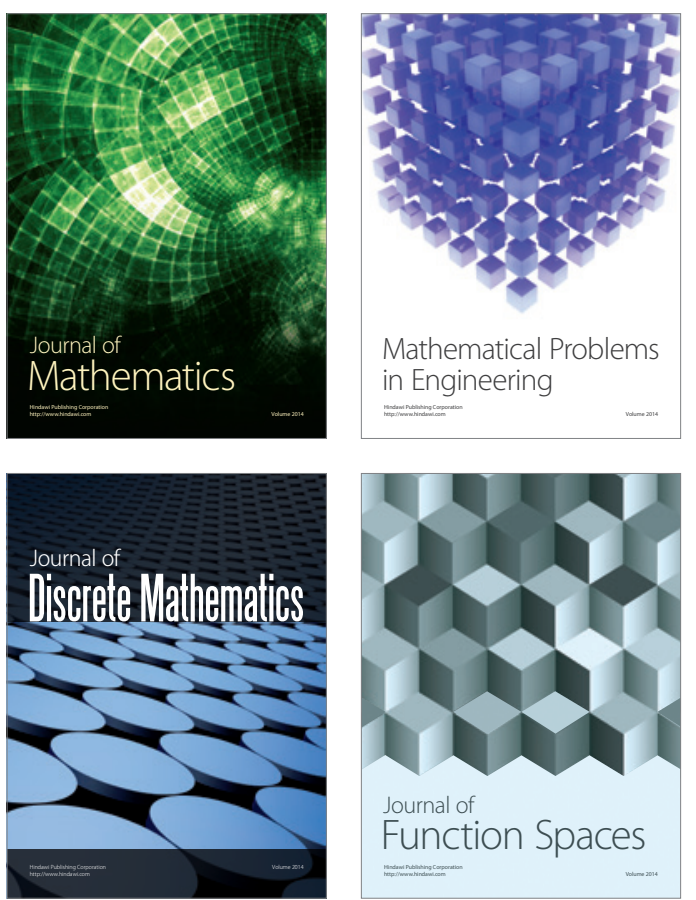

Mathematical Problems in Engineering
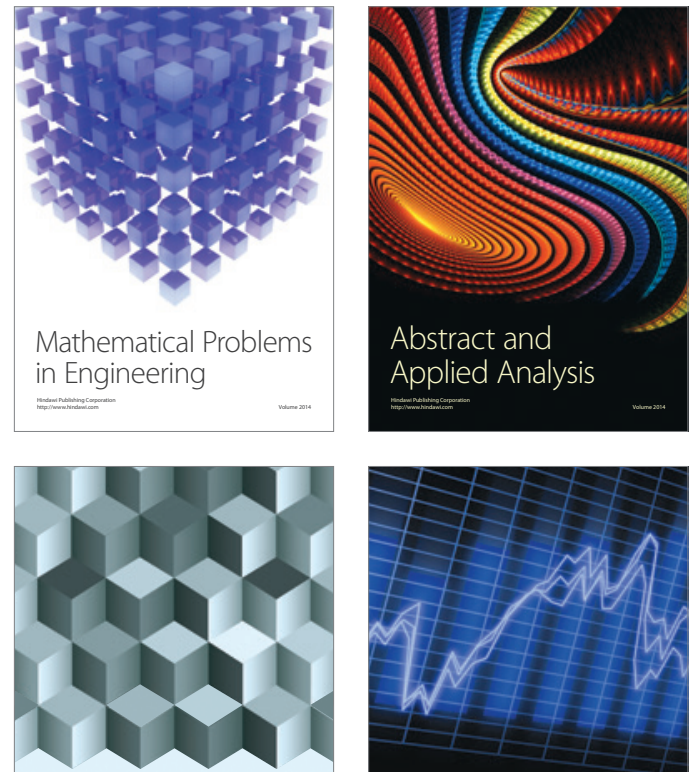

Journal of

Function Spaces

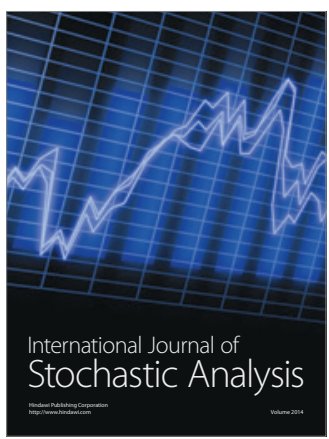

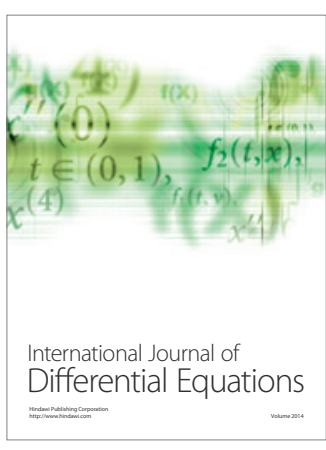
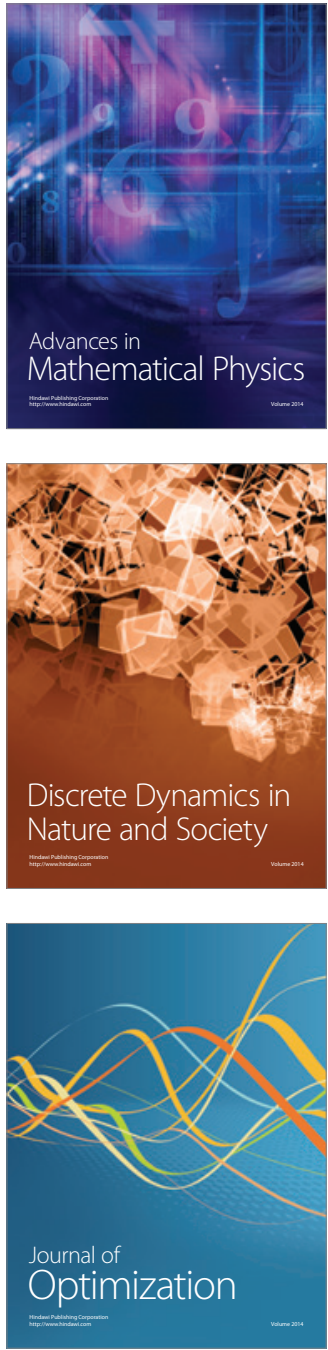